\title{
La diversificación ocupacional y el proceso de la diferenciación socioeconómica de las familias rurales del distrito de Chupaca, 2016
}

\section{Occupational diversification and the socioeconomic differentiation process of rural families in the district of Chupaca, 2016}

\author{
'Tello Yance, Filoter; Rafaele de La Cruz, Mauro \\ Bulege Gutiérrez, Wilfredo \\ Facultad de Sociología, Universidad Nacional del Centro del Perú \\ Email: ftello@uncp.edu.pe
}

\section{Resumen}

El problema de investigación, surgió con el objetivo de analizar la influencia de la diversificación ocupacional en el proceso de la diferenciación socioeconómico de las familias rurales del distrito de Chupaca, 2016; y como objetivos específicos, se plantearon los siguientes: Analizar la influencia de la diversificación ocupacional en el nivel de ingreso socioeconómico de las familias rurales, caracterizar la influencia de la diversificación ocupacional en las condiciones sociales de las familias del distrito de Chupaca y explicar la influencia de la diversificación ocupacional en la estructura de capas y clases sociales entre las familias del distrito de Chupaca. En el desarrollo de la investigación se utilizó el método descriptivo, de análisis y el inductivo. En el proceso de trabajo de campo se aplicó la técnica de la encuesta y su instrumento el cuestionario a 96 informantes. Los datos fueron procesados con la ayuda del programa estadístico SPSS versión 24, los resultados, fueron contrastados con la prueba chi cuadrada para la independencia de criterios, al 95\% de confianza estadística, los resultados evidencian que la mayoría de las familias rurales del distrito Chupaca no tienen diversificación ocupacional (61,5\%), el 30,2 \% poca diversificación y el 8,3\% diversificación moderada; de acuerdo con la diversificación ocupacional, hay diferencias significativas en el ingreso económico $(\mathrm{C}=0,44)$, nivel educativo $(\mathrm{C}=0,388)$, tenencia de servicios básicos de las viviendas $(\mathrm{C}=0,416)$, acceso a algún programa social $(\mathrm{C}=0,394)$, participación en actividades sociales $(\mathrm{C}=0,278)$, organización de fiestas familiares $(\mathrm{C}=$ $0,3)$, estructura de las capas sociales $(C=0,519)$ y estructura de las clases sociales $(\mathrm{C}=0,371)$ de las familias rurales del distrito Chupaca. Se concluyó que la diversificación ocupacional influye significativamente en el proceso de diferenciación socioeconómica de las familias rurales del distrito de Chupaca y; que la diversificación ocupacional influye significativamente en el nivel de ingreso socioeconómico, las condiciones sociales y la estructura de capas y clases sociales entre las familias rurales del distrito de Chupaca.

Palabras clave: diversificación ocupacional, diferenciación socioeconómica, familia rural

\section{Abstract}

The research problem arose with the objective of analyzing the influence of occupational diversification in the socioeconomic differentiation process of rural families in the district of Chupaca, 2016; and as specific objectives, the following were proposed: Analyze the influence of occupational diversification on the level of socioeconomic income of rural families, characterize the influence of occupational diversification on the social conditions of families in the Chupaca district and explain the influence of the occupational diversification in the structure of layers and social classes among the families of the district of Chupaca. In the development of the research, the descriptive, analytical and inductive method was used. In the fieldwork process, the survey technique and its instrument were applied to 96 informants. The data were processed with the help of the statistical program SPSS version 24, the results were compared with the chi square test for the independence of criteria, $95 \%$ of statistical confidence, the results show that the majority of rural families in the Chupaca district do not have occupational diversification (61.5\%), 30.2\% little diversification and $8.3 \%$ moderate diversification; according to the occupational diversification, there are significant differences in economic income $(\mathrm{C}=0.44)$, educational level $(\mathrm{C}$ $=0.388)$, possession of basic housing services $(\mathrm{C}=0.416)$, access to some social program $(\mathrm{C}=0.394)$, participation in social activities $(C=0.278)$, organization of family holidays $(\mathrm{C}=0.3)$, structure of social strata $(\mathrm{C}=0.519)$ and structure of social classes $(\mathrm{C}=0.371)$ of families of the Chupaca district. The conclusions: occupational diversification significantly influences the socioeconomic differentiation process of rural families in the Chupaca district; occupational diversification significantly influences the level of socioeconomic income, social conditions and the structure of layers and social classes among rural families in the Chupaca district.

Keywords: Occupational diversification, socioeconomic differentiation, rural family. 


\section{Introducción}

La diversificación ocupacional, en el ámbito rural del valle del Mantaro en general y, en particular, en el distrito de Chupaca, específicamente en el barrio denominado Vista Alegre, muestra evidencias fehacientes, que nadie puede dudar, desde luego previa verificación y constatación de los hechos, que principalmente cultivaban la papa blanca, el maíz, forrajes para los animales, plantas medicinales en sus huertos y alimentos destinados tanto para el autoconsumo y, una parte de los excedentes de la producción, para el mercado. Esta característica de la agricultura tradicional con la demanda del mercado ha sufrido cambios muy radicales; es decir, actualmente los productores del barrio Vista Alegre, de la parte alta y baja, han modificado la estructura de cultivos tradicionales por el cultivo de 24 tipos de hortalizas y practican la agricultura comercial. La diversificación ocupacional se evidencia por el incremento de actividades y ocupaciones en la unidad familiar de los pequeños y medianos productores. En la actividad agrícola se evidencia la diversificación; es decir, lograron incorporar hortalizas; en la ganadería, se dedican a la crianza de animales mayores y menores; el comercio, el transporte, la artesanía, etc. Lo importante, cabe indicar, que en cada actividad señalada a su vez, existe la diversificación. La actividad agrícola se caracteriza por la diversificación de cultivos, del mismo modo, en la ganadería, con la crianza de animales menores como el cuy, conejo y aves; animales mayores, como vacuno, para la producción leche y como herramienta de labranza, siendo alquilados como yunta, y demás semovientes, como ovinos y cerdos. La diversificación de cultivos comerciales en la actividad agrícola, la crianza en la actividad ganadera y las ocupaciones en las actividades no agrícolas y trae como consecuencia, la evidente diferenciación socioeconómica de las familias y la estructuración de nuevas capas y clases sociales en el campo. A mayor diversificación ocupacional, existe mayor diferenciación socioeconómica y da origen a la estructuración social muy cambiante. Estas características sociales no existían antes. Los niveles de ingreso son muy heterogéneos en función de los ingresos económicos.

\section{Material}

En el desarrollo de la investigación, se utilizaron materiales según la naturaleza del objeto de estudio. La cámara fotográfica para retratar las parcelas familiares con cultivos de diversas hortalizas, el eclímetro para determinar las curvas de nivel, los instrumentos de recojo de datos como los cuestionarios, el uso y manejo de SPSS-versión 25 en el procesamiento de los datos y terminar con la redacción del informe final.

\section{Métodos}

En el proceso de la investigación se utilizó el método científico, método particular, el deductivo y el análisis, tipo de investigación básica.

Nivel de investigación. Según Hernández, Fernández y Baptista (2010, pág.99), el nivel de la investigación es descriptiva, porque caracteriza la diversificación ocupacional en la diferenciación socioeconómica de las familias rurales del distrito de Chupaca.

Diseño de investigación. Según Hernández, Fernández y Baptista (2010, pág.99), el diseño de la investigación es no experimental, transversal o transeccional y correlacional / causal, porque determina la influencia de la diversificación ocupacional en la diferenciación socioeconómica de las familias rurales del distrito de Chupaca, en su ambiente natural, sin realizar ninguna manipulación de la variable independiente, en un solo momento (año 2016).

Técnica de recolección de datos. Fue la encuesta aplicada a familias rurales del Vista Alegre alto y bajo del distrito de Chupaca.

Instrumento de recolección de datos. Fue el cuestionario aplicado a informantes del barrio Vista Alegre, alto y bajo, del distrito de Chupaca.

\section{Resultados}

\section{Diversificación ocupacional de las familias}

Tabla 1

Actividad ocupacional de las familias (Respuestas múltiples, $\mathrm{n}=96$ )

\begin{tabular}{lcc}
\hline \hline \multicolumn{1}{c}{ Actividad ocupacional } & Familias & Porcentaje \\
\hline \hline Agricultura & 72 & 75,0 \\
\hline \hline Ganadería & 31 & 32,3 \\
\hline \hline Comercio & 14 & 14,6 \\
\hline \hline Servicios de transporte & 15 & 15,6 \\
\hline \hline Servicios profesionales & 9 & 9,4 \\
\hline \hline
\end{tabular}

En la Tabla 1 se observa que la mayoría de las familias rurales del distrito Chupaca se dedican a la agricultura (75\%), el 32,3\% a la ganadería, el 14,6\% al comercio, el 15,6\% dan servicios de transporte y el 9,4\% ofrecen servicios profesionales.

El resultado, evidencia la existencia de actividades diversificadas, con predominancia de la agricultura y luego la ganadería, esta última se complementa con el primero. El comercio, el servicio de transporte y los servicios profesionales, son actividades extragrícolas; algunos como el transporte de carga, está vinculado con la actividad agropecuaria, permitiendo transportar insumos como fertilizantes desde las casas comerciales de Huancayo a la chacra y viceversa para trasladar los productos agrícolas y pecuarios al mercado. Este hecho demuestra que los agricultores son ofertantes y están condicionados por la demanda del mercado, ello es una característica propia de una actividad agropecuaria comercial. 
Tabla 2

Cultivos de las familias

(Respuestas múltiples, $\mathrm{n}=96$ )

\begin{tabular}{lcc}
\hline \multicolumn{1}{c}{ Cultivos } & Familias & Porcentaje \\
\hline \hline Papa & 61 & 63,5 \\
\hline \hline Maíz & 58 & 60,4 \\
\hline \hline Zanahoria & 35 & 36,5 \\
\hline \hline Apio & 31 & 32,3 \\
\hline \hline Coliflor & 29 & 30,2 \\
\hline \hline Betarraga & 26 & 27,1 \\
\hline \hline Brócoli & 24 & 25,0 \\
\hline \hline Col & 23 & 24,0 \\
\hline \hline Arvejas & 23 & 24,0 \\
\hline \hline Alfalfa & 23 & 24,0 \\
\hline \hline Ajos & 22 & 22,9 \\
\hline \hline Avena & 22 & 22,9 \\
\hline \hline Trébol & 21 & 21,9 \\
\hline \hline Habas & 19 & 19,8 \\
\hline \hline Ryegrass (heno) & 13 & 13,5 \\
\hline \hline Vicia & 11 & 11,5 \\
\hline \hline Cebada (alcacer) & 10 & 10,4 \\
\hline \hline Perejil & 6 & 6,3 \\
\hline \hline Manzanilla & 6 & 6,3 \\
\hline \hline Alcachofa & 2 & 2,1 \\
\hline \hline Culantro & 2 & 2,1 \\
\hline \hline
\end{tabular}

En la Tabla 2, se descubre que los cultivos predominantes de las familias rurales del distrito Chupaca son papa $(63,5 \%)$ y maíz (60,4\%). Entre el 30 y $40 \%$ de las familias cultivan coliflor $(30,2 \%)$, apio $(32,3 \%)$ y zanahoria $(36,5 \%)$; entre 20 y $30 \%$, trébol $(21,9$ $\%)$, avena $(22,9 \%)$, ajos $(22,9 \%)$, alfalfa (24\%), arvejas $(24 \%)$, col $(24 \%)$, brócoli $(25 \%)$ y betarraga $(27,1 \%)$. Menos del $20 \%$ de las familias cultivan carola $(2,1 \%)$, culantro $(2,1 \%)$, alcachofa $(2,1 \%)$, manzanilla $(6,3 \%)$, perejil $(6,3 \%)$, cebada o alcacer $(10,4 \%)$, vicia $(11,5 \%)$, ryegrass o heno $(13,5 \%)$ y habas $(19,8 \%)$.

La estrategia de la diversificación de algunos cultivos está en función de la demanda del mercado local de la ciudad de Huancayo y el mercado nacional de Lima. Esta realidad indica que los agricultores comerciales están condicionados por el mercado y a dedicarse a una agricultura intensiva moderna, en reemplazo de la agricultura extensiva y tradicional. Esta diversificación de cultivos, también indica ciertos beneficios en los aspectos socioeconómicos como en el nivel educativo, en el acceso a los servicios de salud y el bienestar de las personas y familias, en la calidad de las viviendas y prestigio social.
Tabla 3

Animales que crían las familias

(Respuestas múltiples, $\mathrm{n}=96$ )

\begin{tabular}{lcc}
\hline \hline \multicolumn{1}{c}{ Animales } & Familias & Porcentaje \\
\hline \hline Vacunos & 34 & 35,4 \\
\hline \hline Cuyes & 22 & 22,9 \\
\hline \hline Aves & 9 & 9,4 \\
\hline \hline Ovinos & 5 & 5,2 \\
\hline \hline Cerdos & 3 & 3,1 \\
\hline \hline Conejos & 2 & 2,1 \\
\hline \hline
\end{tabular}

En la Tabla 3, se identifica que el 35,4\% de las familias rurales del distrito de Chupaca crían vacunos, el 22,9\% cuyes, el 9,4\% aves, el 5,2\% ovinos, el 3,1 $\%$ cerdos y el 2,1\% conejos. También diversifican la crianza de diversas especies de ganado, según la importancia económica, de este modo, en la tabla $\mathrm{N}^{\circ}$ 3 , predomina la crianza de vacunos con fines de producción de leche que proveen a la empresa Gloria, lo cual genera un ingreso diario a los productores en comparación a los otros productos agrícolas que generan ingresos por temporadas. La crianza de cuyes y conejos, también tiene importancia económica y alimenticia, los centros gastronómicos de Chupaca y de la ciudad de Huancayo demandan, del mismo modo las otras especies como los ovinos, cerdos y aves de corral.

\section{Ingreso económico de las familias}

\section{Tabla 4}

Formas de tenencia de la tierra de las familias (Respuestas múltiples, $\mathrm{n}=96$ )

\begin{tabular}{lcc}
\hline \hline \multicolumn{1}{c}{ Forma de tenencia } & Familias & Porcentaje \\
\hline \hline Propiedad privada & 72 & 75,0 \\
\hline \hline En arriendo & 15 & 15,6 \\
\hline \hline En hipoteca & 2 & 2,1 \\
\hline \hline Sin tierras & 24 & 25,0 \\
\hline \hline
\end{tabular}

En la Tabla 4 se aprecia que la mayoría de las familias rurales del distrito Chupaca tienen tierras de su propiedad (75\%), el 15,6\% en arriendo y el 2,1\% en hipoteca. El $25 \%$ de las familias no poseen tierras.

En el área de estudio, predomina la forma de propiedad privada, una estructura agraria excesivamente parcelaria con formas tradicionales de tenencia como el arriendo, la hipoteca. El tamaño de las parcelas es muy heterogéneo. Estas tierras agrícolas cuentan con riego permanente que proviene del manantial de Coyllor. La administración del agua está a cargo de la Comisión de Riego del Distrito de Mantaro y al año pagan según la extensión de las tierras. Las tierras agrícolas se han convertido en una mercancía y se manifiesta por la cantidad de compra y venta. 
Tabla 5

Ingreso económico mensual de las familias $(\mathrm{n}=96)$

\begin{tabular}{lcc}
\hline \multicolumn{1}{c}{ Ingreso (soles) } & Familias & Porcentaje \\
\hline \hline$<1000$ & 15 & 15,6 \\
\hline \hline 1000 a 1999 & 27 & 28,1 \\
\hline \hline 2000 a 4999 & 27 & 28,1 \\
\hline \hline 5000 a 9999 & 22 & 22,9 \\
\hline \hline 10000 a + & 5 & 5,2 \\
\hline \hline
\end{tabular}

En la Tabla 5, se detecta que el 28,1\% de las familias rurales del distrito Chupaca tienen ingresos económicos en- tre $\mathrm{S} / .1000 .00$ y S/.1999.00 soles o entre $\mathrm{S} / .2000 .00$ y S/.4999.00 soles mensuales, el 22,9\% entre $S / .5000 .00$ y S/.9999.00 soles, el 15,6\% menos de S/.1000.00 soles y el 5,2\% de $\mathrm{S} / .10000 .00$ mil soles a más.

El resultado del análisis cuantitativo de los resultados de la información, evidencia que las familias de mayor ingreso económico son pocos que las otras capas sociales, la estructuración social en el campo está en función a nivel de ingreso económico y tenencia de bienes como la cantidad de ganado que cría, extensión de tierras, actividades extragrícolas como el comercio, los servicios profesionales y servicios técnicos.

Tabla 5A

Ingreso económico mensual de las familias por diversificación ocupacional $(\mathrm{n}=96)$

\begin{tabular}{|c|c|c|c|c|c|c|c|c|c|c|}
\hline \multirow{3}{*}{$\begin{array}{c}\text { Diversificación } \\
\text { ocupacional }\end{array}$} & \multicolumn{10}{|c|}{ Ingreso económico mensual (soles) } \\
\hline & \multicolumn{2}{|c|}{$<1000$} & \multicolumn{2}{|c|}{1000 a 1999} & \multicolumn{2}{|c|}{2000 a 4999} & \multicolumn{2}{|c|}{5000 a 9999} & \multicolumn{2}{|c|}{$1000 a+$} \\
\hline & $f$ & $\%$ & f & $\%$ & f & $\%$ & f & $\%$ & $\mathbf{f}$ & $\%$ \\
\hline Sin diversificación & 12 & 20,3 & 22 & 37,3 & 17 & 28,8 & 6 & 10,2 & 2 & 3,4 \\
\hline Poca & 3 & 10,3 & 5 & 17,2 & 6 & 20,7 & 12 & 41,4 & 3 & 10,3 \\
\hline Moderada & & & & & 4 & 50,0 & 4 & 50,0 & & \\
\hline
\end{tabular}

En la Tabla 5A y Figura 1, se aprecia que hay diferencias en el ingreso económico de las familias rurales del distrito Chupaca de acuerdo a la diversificación ocupacional, pues el 37,3\% de las familias sin diversificación ocupacional tienen ingresos entre S/.1000.00 y S/.1999.00 soles, el 41,4\% de las familias con poca diversificación tienen ingresos entre $\mathrm{S} / .5000 .00$ y S/.9999.00 soles y el 50 $\%$ de las familias con diversificación moderada, poseen ingresos entre S/.2000.00 y S/.4999.00 soles o entre $\mathrm{S} / .5000 .00 \mathrm{y} \mathrm{S} / .9999 .00$ soles.

El ingreso económico mensual de las familias del niveles de ingreso económico mensual. distrito de Chupaca, están en función a la diversificación ocupacional. Las familias sin diversificación de las actividades económicas obtienen bajos ingresos, carecen de capacidad financiera para invertir en diversificar actividades y por ende los ingresos económicos son de sobrevivencia, tienen una racionalidad no acumulativa; mientras las familias con poca diversificación ocupan otra escala socioeconómica relativamente superior a las familias en proceso de diversificación y las familias de diversificación ocupacional moderada obtienen mayores Figura 1.

Ingreso económico mensual de las familias por diversificación ocupacional

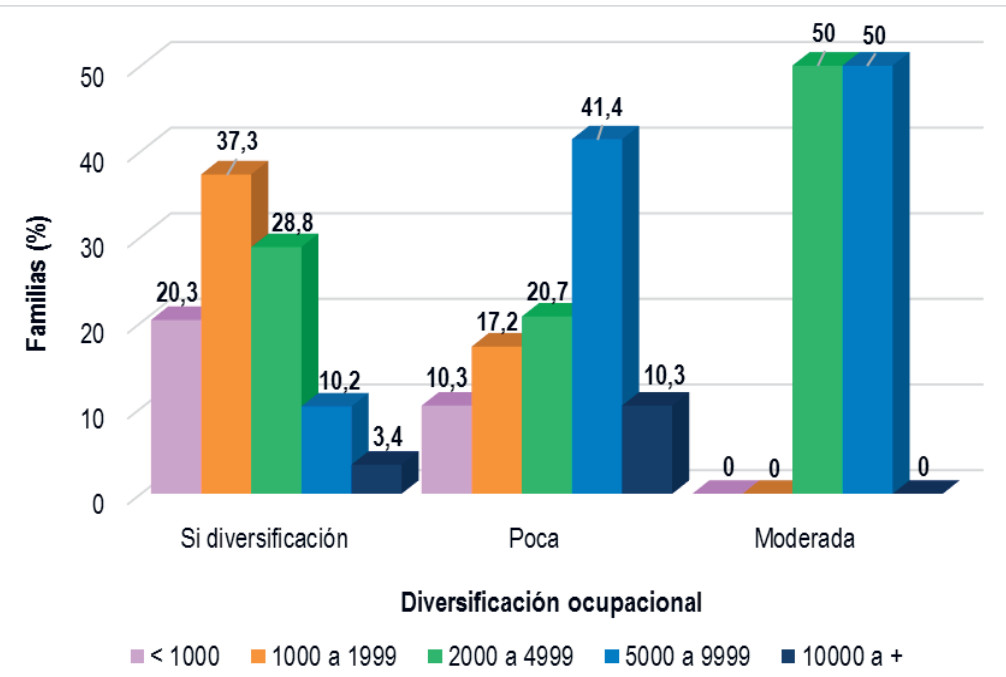

Estos resultados son corroborados con la prueba chi cuadrado para la homogeneidad de muestras, al reportar el valor calculado de 23,098 con el valor $\mathrm{P}$ de 0,003, mayor

que su valor teórico con 8 grados de libertad $(15,51)$ y menor que el nivel de significación usual de 0,05 , respectivamente (Figura 1A, reporte del SPSS V.24). 
Tabla 6

Nivel educativo de las familias por diversificación ocupacional $(\mathrm{n}=96)$

\begin{tabular}{lcccccc}
\hline \hline & \multicolumn{5}{c}{ Nivel educativo } \\
\cline { 2 - 7 } $\begin{array}{c}\text { Diversificación } \\
\text { ocupacional }\end{array}$ & Primaria & \multicolumn{1}{c}{ Secundaria } & \multicolumn{2}{c}{ Superior } \\
\cline { 2 - 7 } & $\mathbf{f}$ & $\%$ & $\mathbf{f}$ & $\%$ & $\mathbf{f}$ & $\%$ \\
\hline \hline Sin diversificación & & & 41 & 69,5 & 18 & 30,5 \\
\hline \hline Poca & 5 & 17,2 & 11 & 37,9 & 13 & 44,8 \\
\hline \hline Moderada & & & 6 & 75,0 & 2 & 25,0 \\
\hline \hline
\end{tabular}

En la Tabla 6 y Figura 2 se observa que hay diferencias en el nivel educativo de las familias rurales del distrito Chupaca según la diversificación ocupacional, pues el 69,5 $\%$ de las familias sin diversificación ocupacional tienen educación secundaria, el 44,8 \% de las familias con poca diversificación tienen educación superior y el $75 \%$ de las familias con diversificación moderada, poseen educación secundaria. Las familias con poca diversificación ocupacional poseen mejores niveles educativos que las familias sin diversificación ocupacional y con diversificación moderada.

Figura 2.

Ingreso económico mensual de las familias por diversificación ocupacional

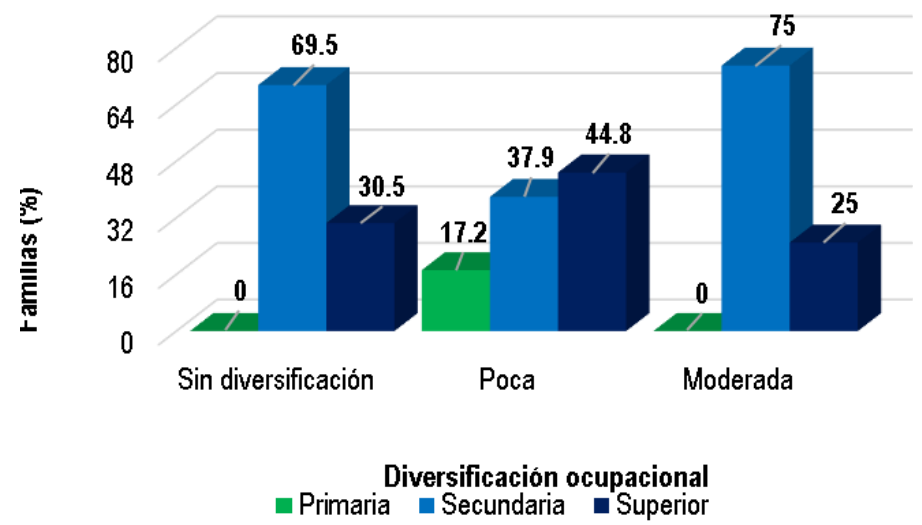

Estos resultados son corroborados con la prueba chi cuadrado para la homogeneidad de muestras, al reportar el valor calculado de 16,447 con el valor $\mathrm{P}$ de 0,002, mayor que su valor teórico con 4 grados de libertad $(9,488)$ y menor que el nivel de significación usual de 0,05 , respectivamente (Figura 4A, reporte del SPSS V.24).

\section{Tabla 7}

Acceso a los servicios de salud de las familias por diversificación ocupacional $(\mathrm{n}=96)$

\begin{tabular}{|c|c|c|c|c|}
\hline \multirow{3}{*}{$\begin{array}{l}\text { Diversificación } \\
\text { ocupacional }\end{array}$} & \multicolumn{4}{|c|}{ Acceso } \\
\hline & \multicolumn{2}{|c|}{ No } & \multicolumn{2}{|c|}{ Si } \\
\hline & $f$ & $\%$ & $f$ & $\%$ \\
\hline Sin diversificación & 4 & 6,8 & 55 & 93,2 \\
\hline Poca & & & 29 & 100 \\
\hline Moderada & & & 8 & 100 \\
\hline
\end{tabular}

Las familias sin diversificación ocupacional acceden en mayor proporción a los servicios de salud; en cambio las familias con poca diversificación ocupacional acuden en poca proporción y finalmente las familias con moderada diversificación ocupacional acuden en pequeña proporción porque prefieren los servicios de salud que ofrecen las clínicas.

En la Tabla 7 y Figura 3, se exhibe que no hay diferencias en el acceso a los servicios de salud de las familias rurales del distrito Chupaca por diversificación ocupacional, pues la mayoría de las familias sin diversificación ocupacional $(93,2 \%)$ y, la totalidad con poca diversificación o diversificación moderada, tienen acceso a los servicios de salud.

\section{Figura 3.}

Acceso a los servicios de salud de las familias por diversificación ocupacional

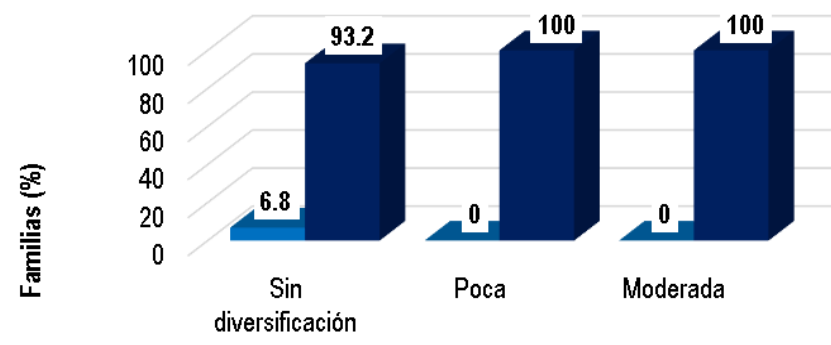

Diversificación ocupacional 
Estos resultados son corroborados con la prueba chi cuadrado para la homogeneidad de muestras, al reportar el valor calculado de 2,618 con el valor P de 0,270, menor que su valor teórico con 2 grados de libertad $(5,991)$ y mayor que el nivel de significación usual de 0,05 , respectivamente (Figura 3A, reporte del SPSS V.24).

\section{Tabla 8}

Tenencia de servicios básicos de las viviendas de las familias por diversificación ocupacional $(\mathrm{n}=96)$

\begin{tabular}{lccccc}
\hline \hline \multirow{2}{*}{$\begin{array}{c}\text { Diversificación } \\
\text { ocupacional }\end{array}$} & \multicolumn{4}{c}{$\begin{array}{c}\text { Tenencia de } \\
\text { servicios básicos }\end{array}$} \\
\cline { 2 - 6 } & \multicolumn{3}{c}{ No } & \multicolumn{3}{c}{ Si } \\
\hline \hline Sin diversificación & $\mathbf{f}$ & $\%$ & $\mathbf{f}$ & $\%$ \\
\hline \hline Poca & 52 & 88,1 & 7 & 11,9 \\
\hline \hline Moderada & 13 & 44,8 & 16 & 55,2 \\
\hline \hline
\end{tabular}

En la Tabla 8 y Figura 4, se descubre que hay diferencias en la tenencia de servicios básicos de las viviendas de las familias rurales del distrito Chupaca de acuerdo con la diversificación ocupacional, pues la mayoría de las viviendas de las familias con baja diversificación ocupacional $(55,2 \%)$ y el $50 \%$ de las familias con diversificación moderada cuentan con los servicios básicos (agua, desagüe y energía eléctrica).

La información evidencia que la diversificación ocupacional, indica en el acceso a los servicios básicos de las familias rurales del distrito de Chupaca; sin embargo, no todos cuentan con todos los servicios, es muy heterogéneo según la ubicación entre el contexto urbano y rural. La mayoría de las familias rurales está en la expansión del casco urbano y acceden a los servicios de saneamiento básico.

Figura 4.

Tenencia de servicios básicos de las familias por diversificación ocupacional

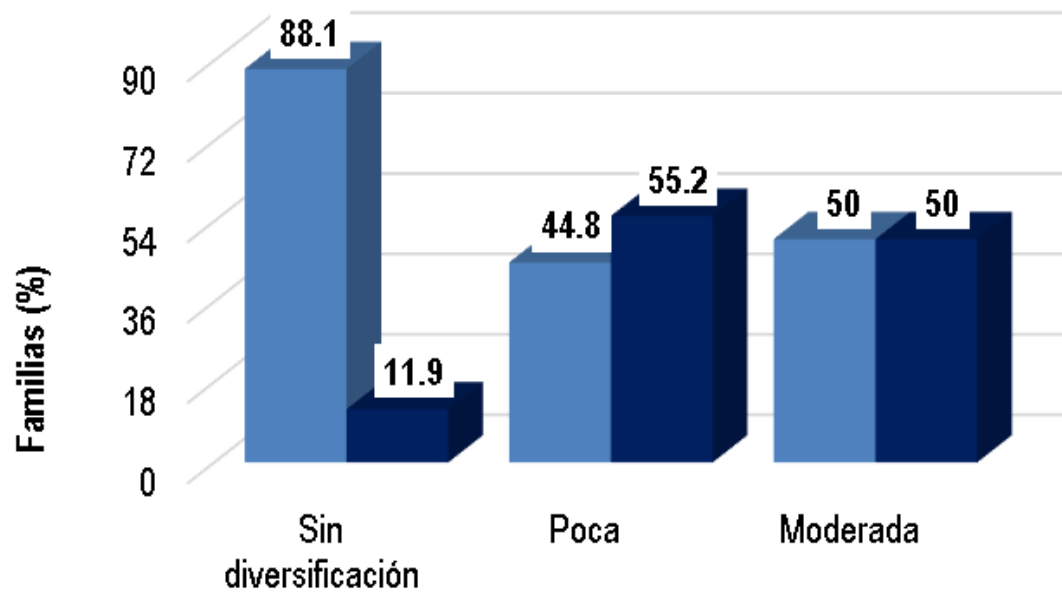

\section{Diversificación ocupacional \\ No $=\mathrm{Si}$}

Estos resultados son corroborados con la prueba chi cuadrado para la homogeneidad de muestras, al reportar el valor calculado de 20,106 con el valor P de 0, mayor que su valor teórico con 2 grados de libertad $(5,991)$ y menor que el nivel de significación usual de 0,05 , respectivamente (Figura 4A, reporte del SPSS V.24).
En la Tabla 9 y Figura 5, se observa que hay diferencias en el acceso a programas sociales de las familias rurales del distrito Chupaca de acuerdo la diversificación ocupacional, pues la mayoría de las familias sin diversificación ocupacional $(83,1 \%)$ y la totalidad con diversificación moderada participan en algún programa social.

Tabla 9

Acceso a algún programa social de las familias por diversificación ocupacional $(\mathrm{n}=96)$

\section{Diversificación ocupa- cional}

Sin diversificación

Poca

Moderada

\section{Participación}

\begin{tabular}{cccc}
\multicolumn{2}{l}{ No } & \multicolumn{2}{c}{ Si } \\
f & $\%$ & f & $\%$ \\
10 & 16,9 & 49 & 83,1 \\
16 & 55,2 & 13 & 44,8 \\
& & 8 & 100
\end{tabular}


Figura 5.

Acceso a algún programa social de las familias por diversificación ocupacional

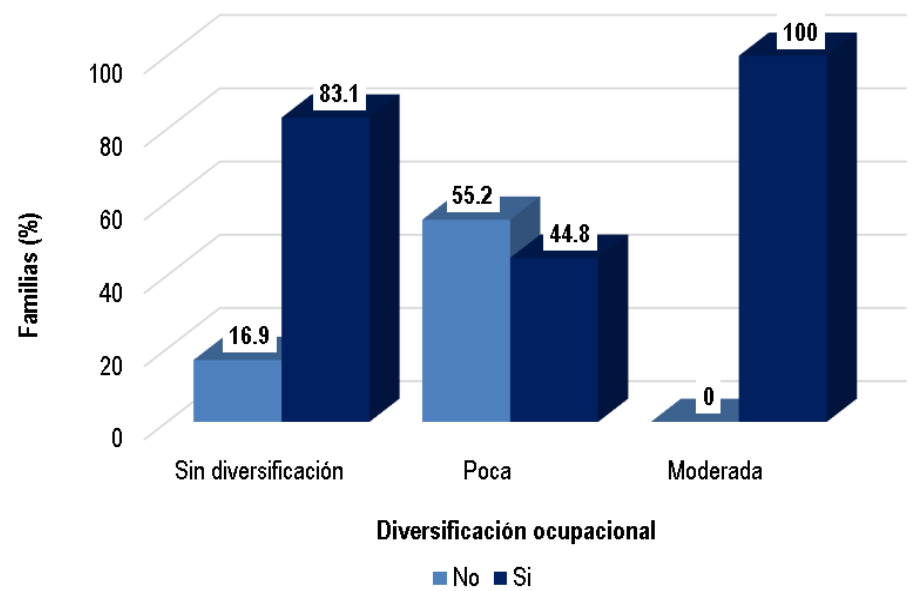

Estos resultados son corroborados con la prueba chi cuadrado para la homogeneidad de muestras, al reportar el valor calculado de 17,626 con el valor $\mathrm{P}$ de 0 , mayor que su valor teórico con 2 grados de libertad $(5,991)$ y menor que el nivel de significación usual de 0,05, respectivamente (Figura 5A, reporte del SPSS V.24).

\section{Tabla 10}

En la Tabla 10 y Figura 6, se aprecia que hay diferencias en la participación en actividades sociales de las familias rurales del distrito Chupaca según la diversificación ocupacional, pues la mayoría de las familias sin diversificación ocupacional (89,8\%), con baja diversificación (69 $\%$ ) y la totalidad con diversificación moderada participan en actividades sociales.

Participación en actividades sociales de las

familias por diversificación ocupacional $(\mathrm{n}=96)$

\begin{tabular}{lcccc}
\hline \multirow{2}{*}{$\begin{array}{c}\text { Diversificación } \\
\text { ocupacional }\end{array}$} & \multicolumn{4}{c}{ Participación } \\
\cline { 2 - 5 } & \multicolumn{3}{c}{ No } & \multicolumn{2}{c}{ Si } \\
\hline \hline Sin diversificación & 6 & 10,2 & 53 & 89,8 \\
\hline \hline Poca & 9 & 31,0 & 20 & 69,0 \\
\hline \hline Moderada & & & 8 & 100 \\
\hline \hline
\end{tabular}

Figura 6.

Participación en actividades sociales de las familias por diversificación ocupacional

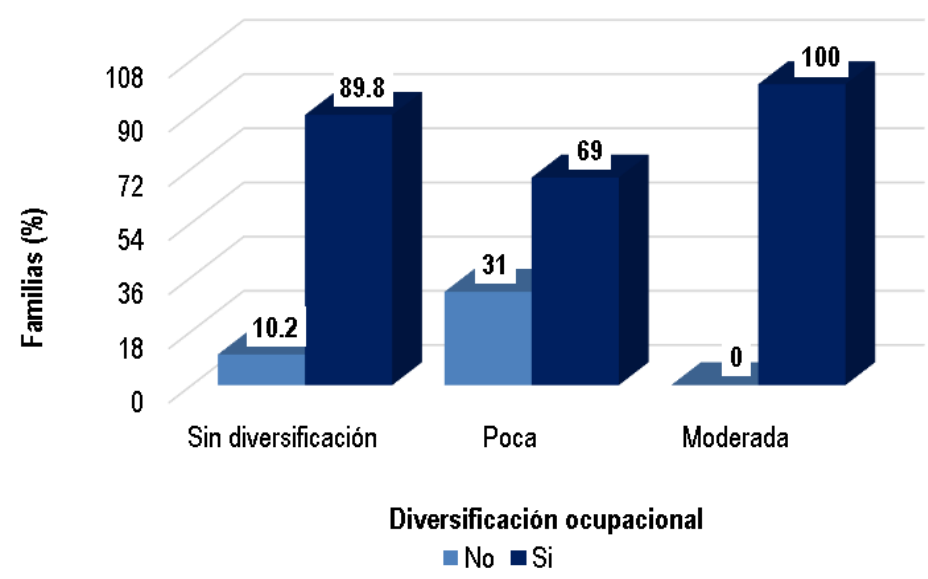

Estos resultados son corroborados con la prueba chi cuadrado para la homogeneidad de muestras, al reportar el valor calculado de 8,037 con el valor $\mathrm{P}$ de 0,018 , mayor que su valor teórico con 2 grados de libertad $(5,991)$ y menor que el nivel de significación usual de 0,05 , respectivamente (Figura 6A, reporte del SPSS V.24). 
Tabla 11

Participación como mayordomo de fiestas de las familias por diversificación ocupacional $(\mathrm{n}=96)$

\begin{tabular}{lrccc}
\hline \hline \multirow{2}{*}{$\begin{array}{c}\text { Diversificación } \\
\text { ocupacional }\end{array}$} & \multicolumn{5}{c}{ Participación } \\
\cline { 2 - 5 } & \multicolumn{3}{c}{ No } & \multicolumn{3}{c}{$\mathbf{S i}$} \\
\hline \hline Sin diversificación & 51 & 86,4 & 8 & 13,6 \\
\hline \hline Poca & 29 & 100 & & \\
\hline \hline Moderada & 8 & 100 & \\
\hline \hline
\end{tabular}

En la Tabla 11 y Figura 7, se aprecia que no hay diferencias en la participación como mayordomo de fiestas de las familias rurales del distrito Chupaca según la diversificación ocupacional, pues la mayoría de las familias sin diversificación ocupacional $(86,4 \%)$ y, la totalidad con baja diversificación y diversificación moderada participan como mayordomo de fiestas.

Estos resultados son corroborados con la prueba chi cuadrado para la homogeneidad de muestras, al reportar el valor calculado de 5,473 con el valor $\mathrm{P}$ de 0,065 , menor que su valor teórico con 2 grados de libertad $(5,991)$ y mayor que el nivel de significación usual de 0,05 , respectivamente (Figura 7, reporte del SPSS V.24).

\section{Figura 7.}

Participación como mayordomo de fiestas de las familias por diversificación ocupacional

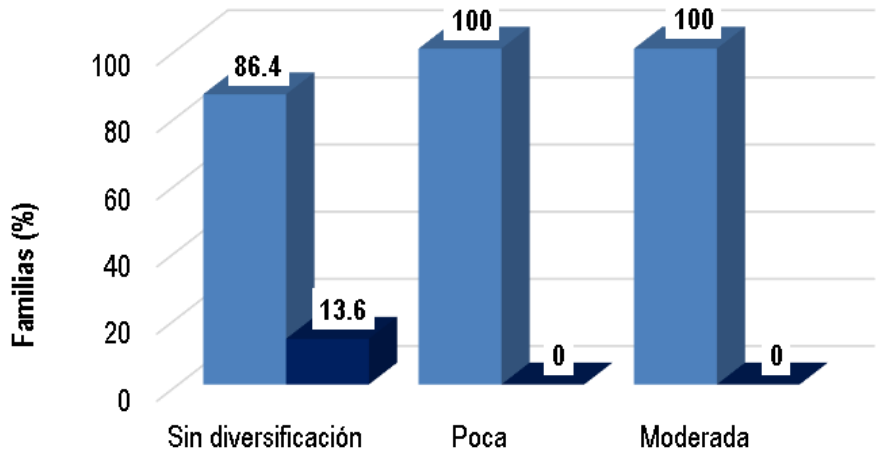

Diversificación ocupacional

$\square$ No $\square \mathrm{Si}$

Tabla 12

Organización de fiestas de las familias por diversificación ocupacional $(\mathrm{n}=96)$

\begin{tabular}{lrcrc}
\hline \hline \multirow{2}{*}{$\begin{array}{c}\text { Diversificación } \\
\text { ocupacional }\end{array}$} & \multicolumn{5}{c}{ Organización } \\
\cline { 2 - 5 } & $\mathbf{f}$ & No & $\mathbf{f}$ & $\%$ \\
\hline \hline Sin diversificación & 38 & 64,4 & 21 & 35,6 \\
\hline \hline Poca & 26 & 89,7 & 3 & 10,3 \\
\hline \hline Moderada & 8 & 100 & & \\
\hline \hline
\end{tabular}

En la Tabla 12 y Figura 8 se detecta que hay diferencias en la organización de fiestas de las familias rurales del distrito Chupaca según la diversificación ocupacional, pues el 35,6\% de las familias sin diversificación ocupacional y el 10,3\% con baja diversificación organizan fiestas familiares.

Figura 8.

Organización de fiestas de las familias por diversificación ocupacional

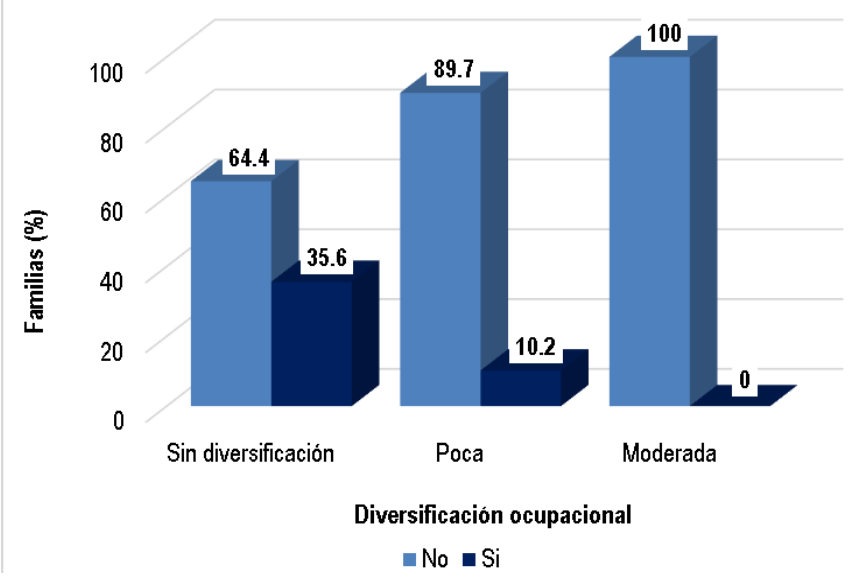


Estos resultados son corroborados con la prueba chi cuadrado para la homogeneidad de muestras, al reportar el valor calculado de 9,520 con el valor $\mathrm{P}$ de 0,009, mayor que su valor teórico con 2 grados de libertad $(5,991)$ y mayor que el nivel de significación usual de 0,05 , respectivamente (Figura 8A, reporte del SPSS V.24).

\section{Estructura de capas y clases sociales de las} familias

\section{Tabla 13}

Estructura de capas sociales de las familias ( $\mathrm{n}=96)$

\begin{tabular}{ccc}
\hline Capa social & Familias & Porcentaje \\
\hline \hline Baja & 17 & 17,7 \\
\hline \hline Media & 64 & 66,7 \\
\hline Alta & 15 & 15,6 \\
\hline \hline
\end{tabular}

En la tabla 13, se aprecia que la mayoría de las familias rurales del distrito Chupaca constituyen la capa social media (66,7\%), el 17,7\% la capa social baja y el 15,6\% la capa alta.
Tabla 13A

Estructura de capas sociales de las familias por diversificación ocupacional $(\mathrm{n}=96)$

\begin{tabular}{lccccccc}
\hline \hline & \multicolumn{5}{c}{ Capa social } \\
\cline { 2 - 7 } $\begin{array}{c}\text { Diversificación } \\
\text { ocupacional }\end{array}$ & \multicolumn{2}{c}{ Baja } & \multicolumn{2}{c}{ Media } & \multicolumn{2}{c}{ Alta } \\
\cline { 2 - 7 } & f & $\%$ & $\mathbf{f}$ & $\%$ & $\mathbf{f}$ & $\%$ \\
\hline \hline Sin diversificación & 17 & 28,8 & 40 & 67,8 & 2 & 3,4 \\
\hline \hline Poca & & & 16 & 55,2 & 13 & 44,8 \\
\hline \hline Moderada & & & & 8 & 100 & & \\
\hline \hline
\end{tabular}

En la Tabla 13A y Figura 9, se observa que hay diferencias en las capas sociales de las familias rurales del distrito Chupaca de acuerdo con la diversificación ocupacional, pues la mayoría de las familias sin diversificación ocupacional $(67,8 \%)$, con baja diversificación $(55,2 \%)$ y la totalidad con moderada diversificación constituyen la capa social media y, el 44,8 \% de las familias con poca diversificación conforman la capa social alta.

Figura 9.

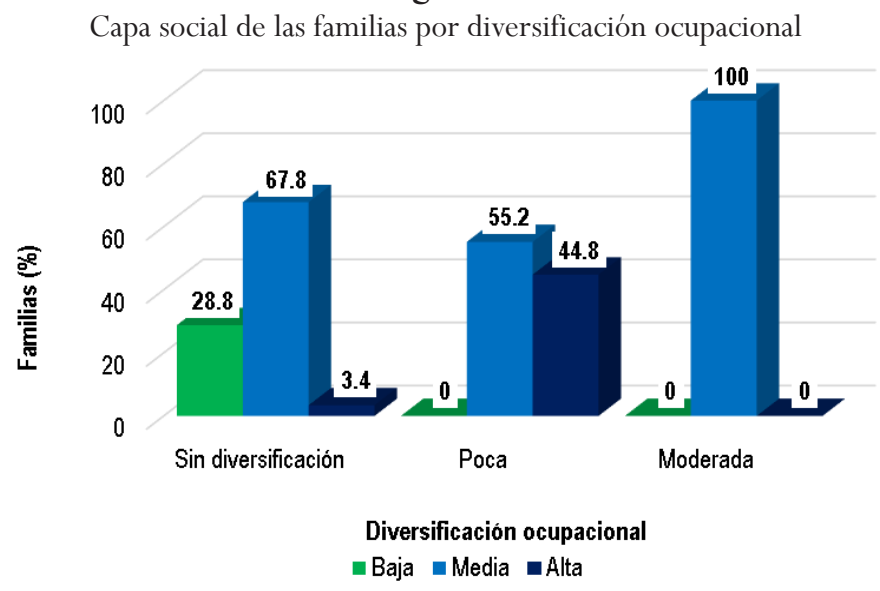

Estos resultados son corroborados con la prueba chi cuadrado para la homogeneidad de muestras, al reportar el valor calculado de 35,311 con el valor $\mathrm{P}$ de 0 , mayor que su valor teórico con 4 grados de libertad $(9,488)$ y menor que el nivel de significación usual de 0,05, respectivamente (Figura 9A, reporte del SPSS V.24).

En la Tabla 14, se observa que la mayoría de las familias rurales del distrito Chupaca constituyen la clase social peón agrícola (74\%) y el $26 \%$ la clase burguesía rural.

En la Tabla 14A y Figura 10, se constata que hay diferencias en las clases sociales de las familias rurales del distrito Chupaca de acuerdo con la diversificación ocupacional, pues la mayoría de las familias sin diversificación ocupacional son peones agrícolas $(83,1$ $\%$ ), la mayoría de las familias con baja diversificación constituyen la burguesía rural y la totalidad de las familias con diversificación moderada, son peones agrícolas.

\section{Tabla 14A}

Estructura de clases sociales de las familias por diversificación ocupacional $(\mathrm{n}=96)$

\begin{tabular}{lcccc}
\hline \hline \multirow{2}{*}{$\begin{array}{c}\text { Diversificación } \\
\text { ocupacional }\end{array}$} & \multicolumn{4}{c}{ Clase social } \\
\cline { 2 - 5 } & \multicolumn{2}{c}{ Peón agrícola } & \multicolumn{3}{c}{ Burguesía rural } \\
\cline { 2 - 5 } & $\mathbf{f}$ & $\%$ & $\mathbf{f}$ & $\%$ \\
\hline \hline Sin diversificación & 49 & 83,1 & 10 & 16,9 \\
\hline \hline Poca & 14 & 48,3 & 15 & 51,7 \\
\hline \hline Moderada & 8 & 100 & & \\
\hline \hline
\end{tabular}


Figura 10.

Clase social de las familias por diversificación ocupacional

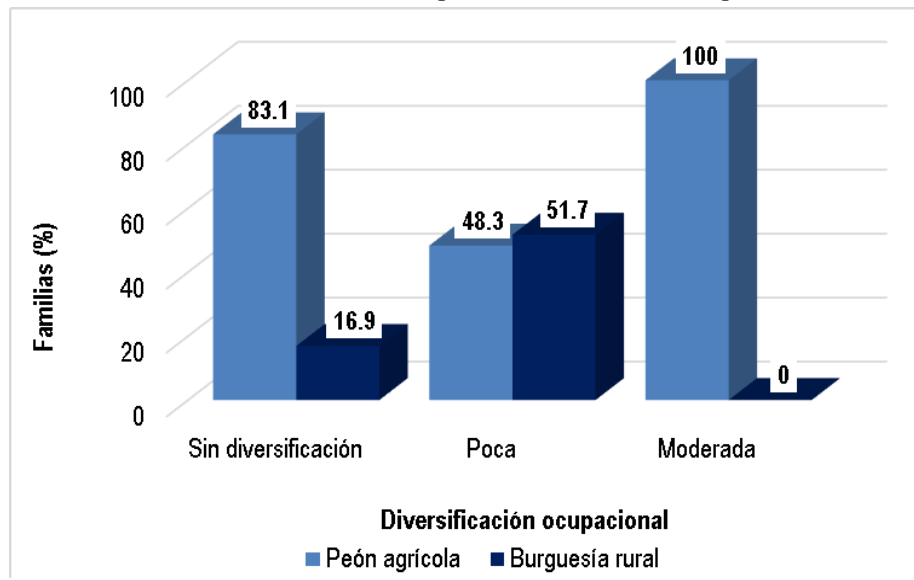

Estos resultados son corroborados con la prueba chi cuadrado para la homogeneidad de muestras, al reportar el valor calculado de 15,281 con el valor $\mathrm{P}$ de 0, mayor que su valor teórico con 2 grados de libertad $(5,991)$ y menor que el nivel de significación usual de 0,05, respectivamente (Figura 10A, reporte del SPSS V.24).

\section{Discusión}

En esta fase, se compara los resultados obtenidos de investigadores de ámbito internacional y nacional con los hallazgos del área de estudio.

Zoomers (1998), quién realizó estudios sobre la diversificación ocupacional y el proceso de la diferenciación socioeconómica de las familias rurales en Bolivia, sostiene, que es una estrategia de lucha contra la pobreza como parte de un proceso autónomo de alternativas, aprovechando las oportunidades de la demanda del mercado. En Chupaca, esta iniciativa similar entre las familias en el área de estudio está en proceso, hasta la fecha, de 96 familias encuestas, sólo el 35 \% practica la diversificación y se evidencia que el nivel de ingreso se incrementa y la influencia no es directa. La investigación contribuye al dar cuenta los cambios de la lógica interna del caso de las familias rurales en Vista Alegre, alto y bajo, como parte de un proceso de cambios internos influenciado por factores externos, principalmente por la demanda de mercado, ellos ofertan hortalizas de diferentes especies y variedades. La multiactividad es una de las características de la diversificación ocupacional y el proceso de diferenciación socioeconómica, las familias campesinas tradicionales se dedicaban sólo a la pequeña agricultura de autosubsistencia y luego se transformaron en economías mercantiles y actualmente se dedican a la agricultura y ganadería comercial y complementan con actividades extra-agrícolas, destacándose el comercio, servicio de transporte, servicios profesionales y alquiler de maquinaria agrícola.

García (2011), en su libro Proceso de acumulación de capi- tal en campesinos. El caso de los horticultores bolivianos migrantes asentados en Buenos Aires (Argentina), declara que el proceso de capitalización de campesinos se asocia, en el mejor de los casos, con el surgimiento de una pequeña burguesía rural, adoptándose como patrón de referencia al capitalismo urbano-industrial, entendiéndose, por lo tanto, innecesario el estudio de los mecanismos de su funcionamiento específico de las actividades agropecuarias. La realidad demuestra que la capitalización de campesinos es un proceso más complejo de lo que se piensa. Para Schneider (2016), el objetivo de este trabajo consiste en presentar argumentos a favor de las potencialidades de la pluriactividad como una de las formas de promover estrategias sostenibles de diversificación de los modos de vida de las familias rurales de Brasil y ampliar sus condiciones de reproducción social y económica. Se cree que, a través de la pluriactividad, las familias de agricultores pueden establecer iniciativas de diversificación de sus ocupaciones interna y externamente a la unidad de producción; así como, aumentar las fuentes y las formas de acceso a los ingresos.

\section{Conclusiones}

- La diversificación ocupacional influye significativamente en el proceso de diferenciación socioeconómica, en el nivel de ingreso económico, en las condiciones sociales y en la estructuración de nuevas capas y clases sociales entre las familias rurales del distrito de Chupaca.

- La diversificación ocupacional influye significativamente en el nivel de ingreso socioeconómico de las familias rurales del distrito de Chupaca (coeficiente de contingencia de $\mathrm{C}=0,44)$.

- La diversificación ocupacional influye significativamente en las condiciones sociales de las familias rurales del distrito de Chupaca, fundamentalmente en el nivel educativo $(\mathrm{C}=0,388)$, tenencia de servicios básicos de las viviendas $(\mathrm{C}=0,416)$, acceso 
a algún programa social $(C=0,394)$, participación en actividades sociales $(\mathrm{C}=0,278)$ y organización de fiestas $(C=0,3)$.

- La diversificación ocupacional influye significativamente en la estructura de capas y clases sociales entre las familias rurales del distrito de Chupaca, con coeficientes de contingencia de 0,519 y 0,371 , respectivamente.

- La diversificación ocupacional influye significativamente en el proceso de diferenciación socioeconómica, en el nivel de ingreso socioeconómico, en las condiciones sociales y la estructura de capas y clases sociales entre las familias rurales del distrito de Chupaca.

\section{Recomendaciones}

- Promover iniciativas de emprendimiento, aprovechando las capacidades y potencialidades locales de los agricultores hacia la diversificación de actividades productivas y de servicios aprovechando las oportunidades de demanda; orientada a la mejora sostenible de las condiciones socioeconómicas, para asegurar las condiciones: sociales, calidad de vivienda, salud, educación, infraestructura y tecnologías productivas en beneficio de las familias rurales del distrito de Chupaca, del valle del Mantaro y de la región Junín.

- Organizar la asociatividad de pequeñas y microempresas agropecuarias de servicios múltiples rurales con apoyo financiero, técnico, normativo y con acceso a oportunidades de mercado nacional y agroexportación.

\section{Referencias bibliográficas}

García, M. (2011). Proceso de acumulación de capital en campesinos. El caso de los horticultores bolivianos de Buenos Aires (Argentina), Cuadernos de desarrollo rural 8, (66): 47-70.

Schneider, S. (2016). La contribución de la pluriactividad para las politicas públicas de desarrollo rural: una mirada desde el Brasil.

Sierra Bravo, R. (2005). Técnicas de investigación social, teorías y ejercicios. Madrid- España, editorial THOMSON.

Zoomers, A. (1998). Estrategias campesinas: algunas consideraciones teóricas y conceptuales. Sucre- Bolivia, editorial CEDLA. 